\title{
EFFECT OF OXIDIZING CONDITIONS ON ACCELERATED ELECTROLYTIC CORROSION TESTS
}

\author{
By Henry S. Rawdon and W. A. Tucker
}

ABSTRACT

Accelerated electrolytic corrosion tests were made on sheet copper and nickel in a sodium-chloride solution. Both anode and cathode were of the same metal, and the applied emf was relatively very low; the results are expressed as loss-ofweight-potential curves. The effect of oxidizing conditions was studied by surrounding the cell by an atmosphere of oxygen or nitrogen and/or dissolving the same gas in the electrolyte. The results clearly show the accelerating effect of oxygen on corrosion by reducing the potential necessary for a given corrosion rate. The results also have a distinct bearing on the theory of underwater corrosion as affected by conditions of differential oxidation within the solution.

\section{CONTENTS}

I. Introduction

II. Materials and method

1. Materials_...

2. Method

III. Results

IV. Discussion _.

V. Summary

\section{INTRODUCTION}

An electrolytic method based on the assumption that corrosion is essentially electrolytic in its nature - that is, it involves two distinct processes, anodic and cathodic-was recently described ${ }^{1}$ as a logical procedure for studying corrosion phenomena. In this report the principles were outlined which, it was felt, must be observed if results consistent with "natural" corrosion results are to be obtained.

Somewhat later, this proposed method was used in a study of the comparative merits of a number of different kinds of corrosion tests carried out on the same metals in the same kind of solution. ${ }^{2} \mathrm{Al}-$ though the data secured were not extensive enough to warrant a

1 W. Blum and H. S. Rawdon, Principles of Electrolytic Studies of Corrosion, Trans. Am. Electrochem. Soc., 5?, p. 403; 1927.

${ }^{2}$ H. S. Rawdon and E. C. Groesbeck, The Effect of the Testing Method on the Determination of Corrosion Resistance, B. S, Tech. Paper No. 367; 1928. 
definite recommendation concerning the use of the accelerated electrolytic test in preference to any other, the agreement, on the whole, of the results of this test with those of simple immersion was as good as was obtained with the other methods used. However, the results obtained in a few tests in which the corrodent had been saturated with nitrogen as a means of expelling any oxygen which might be present in the solution before the corrosion test was carried out, suggested that the results obtained in an accelerated electrolytic corrosion test may be markedly influenced by the condition of the solution with respect to the presence of oxygen or other gases.

The present study was carried out primarily with the aim of studying this effect in greater detail in order to show whether or not a careful control of the conditions, with respect to oxidation, under which the accelerated electrolytic test is carried out is necessary. Incidentally, it was also hoped that some information on the general subject of the effect of aeration on "submerged corrosion" (in particular, "anodic corrosion") might be gained.

Studies recently carried out by Tödt, ${ }^{3}$ the results of which were available to the authors only after the present tests had been nearly completed, show that in the case of "couples" of dissimilar metals immersed in an electrolyte the magnitude of the current set up in any particular case is dependent in large measure upon the condition of the solution with respect to the access of oxygen (air).

\section{MATERIALS AND METHOD}

\section{MATERIALS}

Commercial copper and nickel in sheet form were the two metals used throughout. The specimens were cut from the same sheet as was used in the previous study. ${ }^{4}$ A normal solution of sodium chloride was used as the corrodent throughout. In the experiments which involved the use of oxygen or nitrogen, gas of ordinary commercial purity was used.

\section{METHOD}

The method, which has already been described in detail, ${ }^{5}$ consisted essentially in determining the loss of weight of a specimen of sheet metal used as the anode of an electrolytic cell with a cathode of the same material "facing" the anode on each side at a definite distance, $2.5 \mathrm{~cm}$ (1 inch). The assembled "cell" is shown in Figure 1. For purposes of reference, Figure 2 has been reproduced here from the previous report and shows a diagram of the complete set-up. In the

${ }^{3}$ F. Tödt, Corrosion and Residual Currents, Korrosion und Metallschutz, 3, 37-38; 1928; Zeit. Elektrochem, 34, pp. 586-591; 1928.

- See footnote 1, p. 375 .

s See footnote 1, p. 375 . 
B. S. Journal of Research, RP101

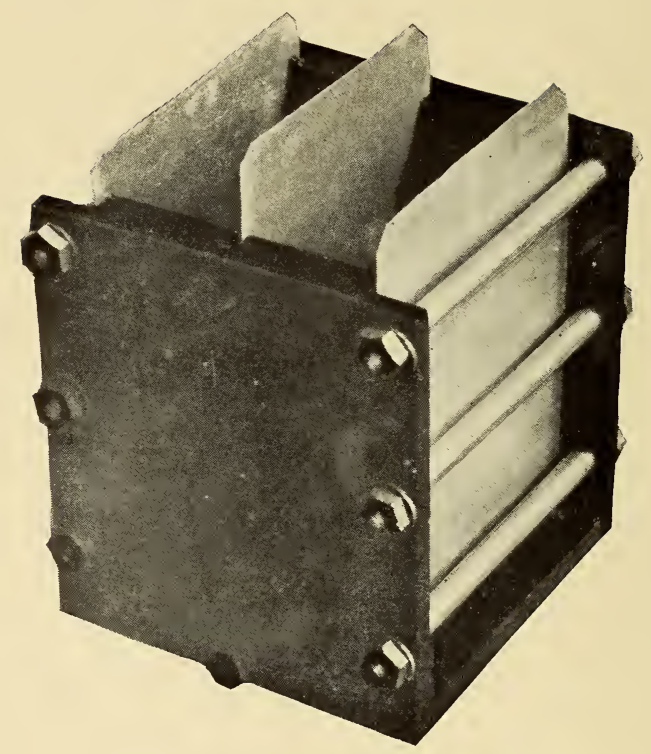

Figure 1.- "Cell" used in the test 
previous work the cathode plates formed the ends of the cell and, of necessity, projected above the surface of the solution used, the volume of which $\left(125 \mathrm{~cm}^{3}\right)$ was kept constant throughout the tests. In the present series of tests glass ends were used in the cell in order to permit the use of totally submerged specimens as well as partially submerged specimens. Only the latter were used in the previous tests. In all cases in which the specimens were fully submerged the surface area of the specimen was equal to that wet by the solution in the tests involving partial immersion. This was $25 \mathrm{~cm}^{2}$.

The sodium-chloride solution was, for nearly all of the tests, prepared from distilled water which had been freshly boiled in order to

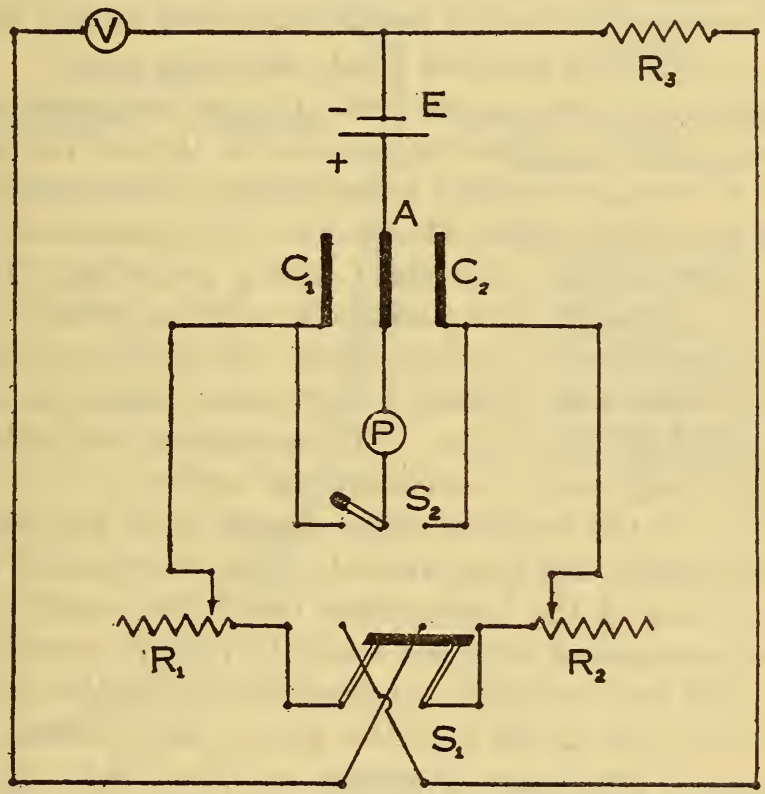

Figure 2.-Electrical "set-up"

render it gas free. In a few cases, however, the solution was intentionally prepared from distilled water with which no precautions had been taken to render it free from air. In a number of other cases the sodium-chloride solution, after being prepared from gas-free distilled water, was "charged" with oxygen or nitrogen by bubbling the gas through it. When it was desired to maintain an atmosphere of oxygen or of nitrogen above the solution the cell was inclosed beneath a bell jar through which a gentle stream of oxygen (or nitrogen) was passed and allowed to escape into the surrounding atmosphere.

In determining the effect of each variable studied a number of runs (usually four) was taken, the voltage applied being increased 
for each succeeding "run" and the results expressed in the form of loss-of-weight-potential curves. As a basis of reference, curves have been included in this report which show the current densities produced at each potential under corresponding conditions. To facilitate comparison with the loss-of-weight curves, these current densities have been expressed in terms of the equivalent weights of the metals by assuming 100 per cent anode efficiency (as $\mathrm{Cu}^{\mathrm{I}}$ and $\mathrm{Ni}^{\mathrm{II}}$ ). In short, these curves show the loss of weight calculated in accordance with Faraday's law for the current resulting from the applied potential, and the departure of the curves showing the observed losses of weight from these curves represents the departure from 100 per cent anode efficiency for the currents measured. For brevity, hereinafter, the curve showing the loss of weight computed in this way will be referred to as the " 100 per cent anode efficiency curve."

All of the tests were carried out at room temperature, and no attempt was made to agitate the solution in the cell during the run.

In order to obtain complete submergence of the specimens-that is, to avoid any surface effect whatsoever-the specimens to be totally submerged were cut from the sheet with a projecting "tail." This tail, which was covered with paraffin in order to protect it from the action of the solution, projected above the surface of the solution several centimeters and afforded a convenient means for making the necessary electrical connection. The specimens were submerged so that the upper edge was $0.5 \mathrm{~cm}$ below the surface.

The surface of the specimens was cleaned with fine emery paper, rubbed with pumice, and then washed. They were sealed with paraffin into the slots of the hard-rubber box before starting a "run." The anode was washed with hot water to remove paraffin after the run, dried, and then weighed. It should be noted that both sides of the anode were used in the test; this gave a total surface of $50 \mathrm{~cm}^{2}$. Each run was of two hours' duration, and the loss for 24 hours was calculated on the basis of this. In practically all of the tests the voltage was observed to fall gradually, and it was maintained at the initial value only by lowering slightly the resistance of the circuit at intervals during the run.

\section{RESULTS}

The different variables given consideration in the various tests carried out were as follows:

1. Degree of immersion-total, partial.

2. Condition of the solution with respect to dissolved gas-containing dissolved air, freed from dissolved air, containing oxygen, containing nitrogen.

3. Atmosphere above the solution-air, oxygen, nitrogen. 
The results obtained are summarized graphically in Figures 3 to 8, inclusive, as loss-of-weight-potential curves. Curves showing 100 per cent anode efficiency have also been given as a convenient means of comparison and reference. These computed losses on the basis of Faraday's law were based on the assumption that copper passes into solution as $\mathrm{Cu}^{\mathrm{I}}$ and nickel as $\mathrm{Ni}^{\mathrm{II}}$.

From the results given in Figures 3 and 4 it will be seen that no very marked differences resulted from total immersion and from partial immersion in solutions containing some dissolved air as compared with similar tests in solution which had been freed from dissolved air. The curves followed rather closely throughout most of

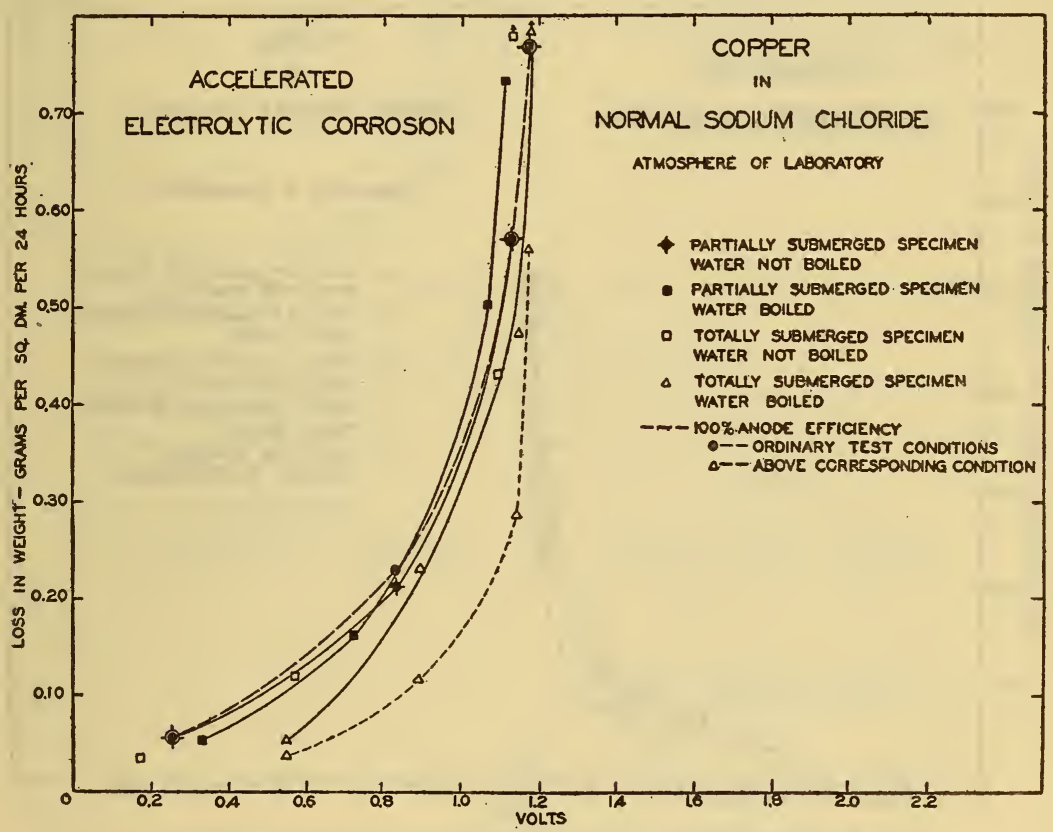

FIGURE 3.-Results with copper-ordinary conditions

their course that of the curve for 100 per cent anode efficiency for ordinary test conditions. The greatest divergence occurred with small anode losses; that is, low current densities. The most marked divergence from the curve showing 100 per cent anode efficiency was in the case of total immersion in a gas-free solution. It should be noted, however, that the curve showing 100 per cent anode efficiency under these conditions does not coincide with the other curve of this kind. In this particular case the observed losses were apparently greater than those indicated by the 100 per cent anode efficiency curve. The reason for this apparent anomaly is discussed later in the report. It should be noted that the current density for a given voltage - that is, the 100 per cent anode efficiency curves-varied some- 
what according to the condition of the solution. For each curve, therefore, showing the relation between the applied voltage and the observed corrosion loss (loss of weight), a corresponding curve showing 100 per cent anode efficiency for the current produced by the applied emf may be drawn. In the following figures such curves have been drawn for those conditions which differ most widely.

It may be well to point out at this point that a fundamental assumption of the electrolytic corrosion test is that the corrodibility of a metal tested in this way is indicated by the potential necessary to produce a certain current density and, hence, a loss of weight. If the loss of weight is approximately that calculated by Faraday's law

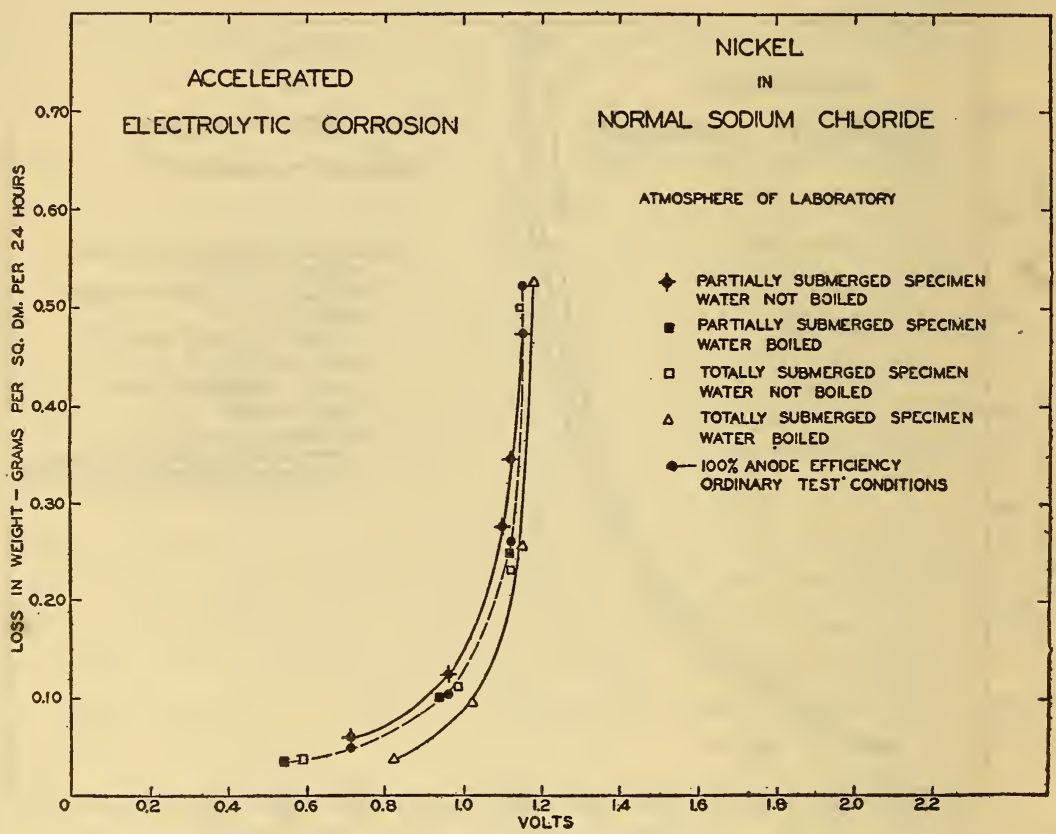

FigURe 4.-Results with nickel-ordinary conditions

on the basis of the observed current, the "anode efficiency" is practically 100 per cent. The indications of the electrolytic test in such cases are not nearly so valuable as when the corrosion is very much less; that is, when the corroding anode shows a tendency to become passive. In those cases in which the anode corrosion is apparently greater than 100 per cent-that is, greater than the loss calculated by Faraday's law for the observed current-it is obvious that some other explanation must be sought. Thus, for example, it may be that the measured current does not represent the entire current which is contributing to the anode corrosion loss. "Parasitic" currents within the cell itself resulting from some nonuniform conditions often appear to be responsible. 


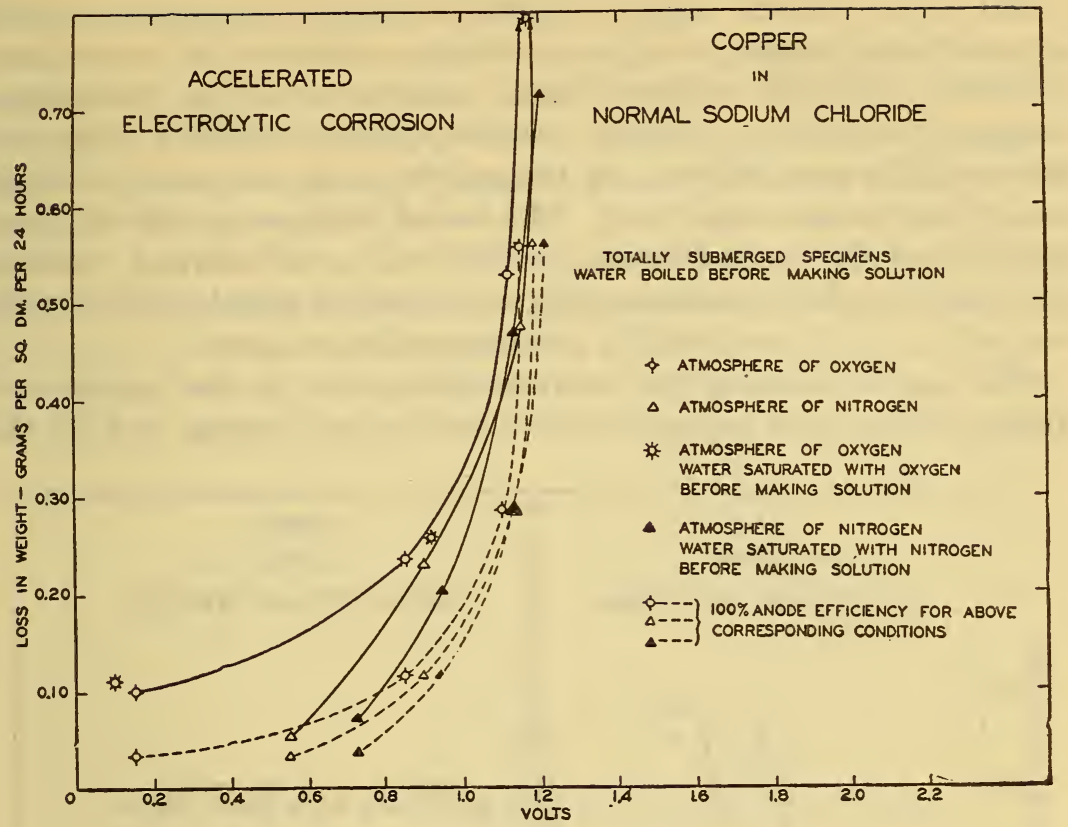

Figure 5.-Results with copper

Specimens were completely immersed and an atmosphere of oxygen or of nitrogen was used

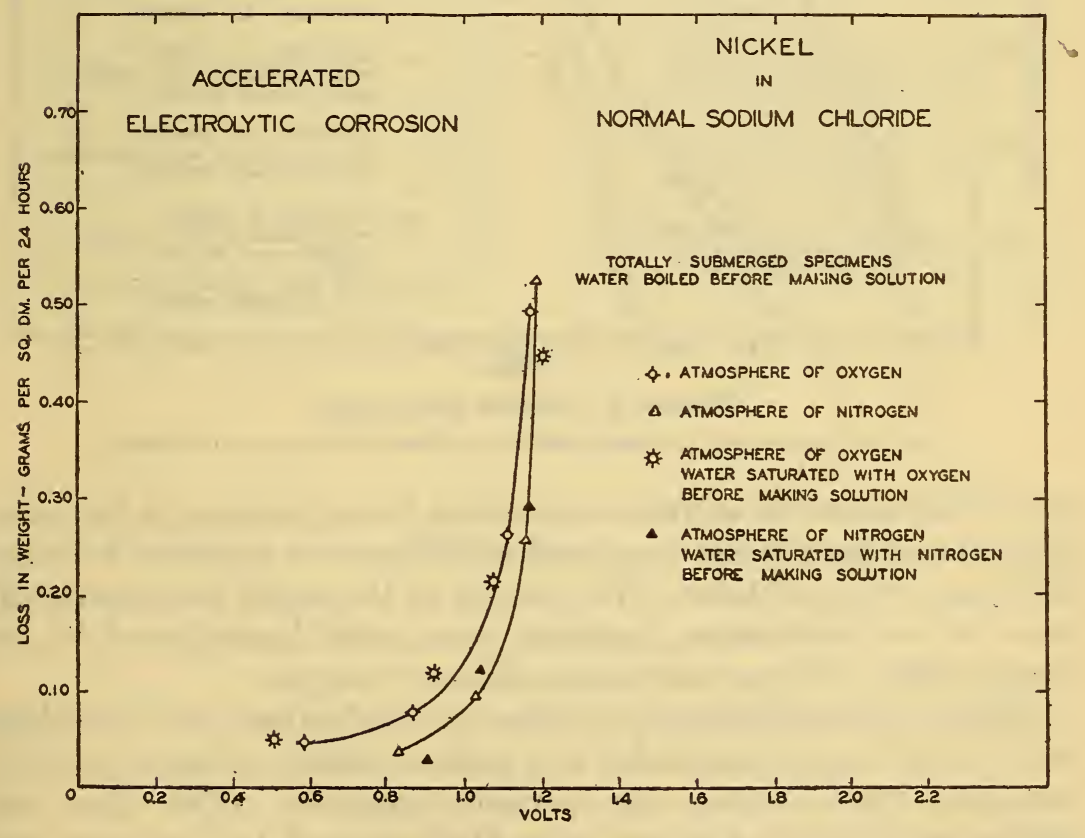

FIgURE 6.-Results with nickel

Conditions as in Figure 5 
The curves given in Figures 5 and 6, for totally immersed specimens with the introduction of oxygen or nitrogen, either as the atmosphere overlying the plain sodium-chloride solution or as the atmosphere overlying the sodium-chloride solution charged with the same gas, did not differ markedly among themselves except for small corrosion losses (low current densities). The use of nitrogen as the overlying atmosphere above the solution or dissolved in the solution rendered the metal somewhat more resistant to corrosive attack than was the case when oxygen was used in a corresponding manner.

The curves showing the corrosion behavior in the presence of oxygen, either with oxygen as the overlying atmosphere or with this

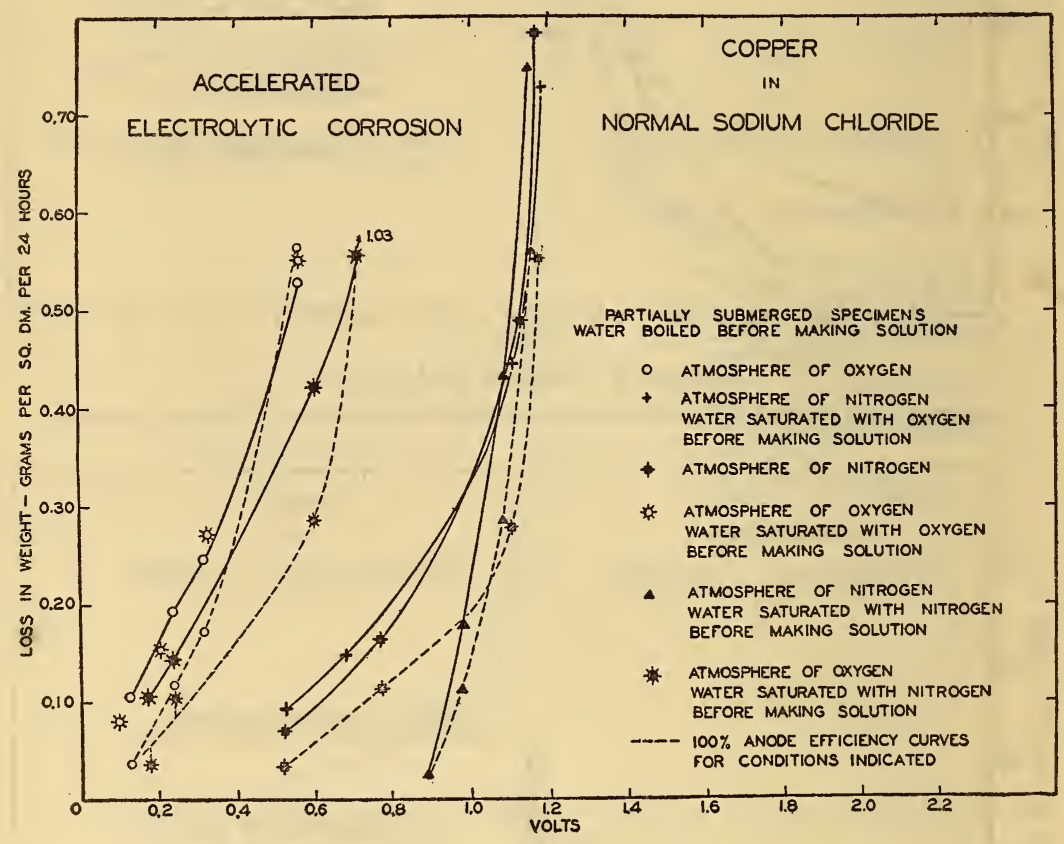

Figure 7.-Results with copper

Specimens were partially immersed; conditions, otherwise, the same as in Figure 5

gas dissolved in the solution in addition to its presence in the overlying atmosphere, indicate no marked difference in corrosion behavior for these two conditions. The attack of the metal was apparently more severe in all cases, however, than would be indicated by the corresponding 100 per cent anode efficiency curves.

The most marked effect of oxygen in accelerating the electrolytic corrosion of copper and nickel in a sodium-chloride solution occurred when the specimen was only partially immersed. This effect was rather pronounced in all cases in which part of the specimen was exposed to an oxygen atmosphere, regardless of whether the solution was initially "gas free," saturated with oxygen, or saturated with 
nitrogen. Figures 7 and 8 show this effect. On the other hand, specimens partially immersed in an oxygen-saturated solution and having the upper part exposed in a nitrogen atmosphere behaved practically identically with specimens partially immersed in a "gasfree" solution with an overlying nitrogen atmosphere. In all cases in which nitrogen was used as the enveloping atmosphere or, in addition, for saturating the solution the corrosion behavior of the partially immersed specimens was quite similar to that of the corresponding totally immersed specimens. (Figs. 5 and 6.)

In Figure 9 are given typical curves showing the relation between voltage and current density for some of the runs made with copper.

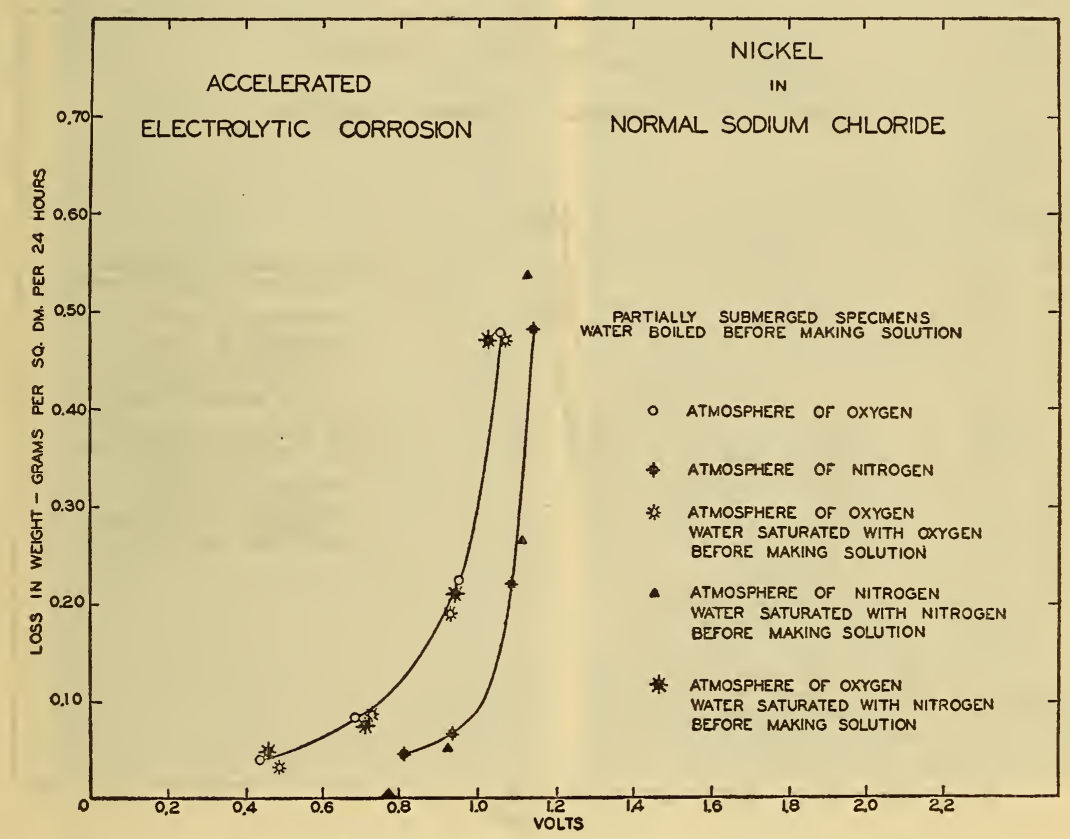

FIGURE 8.-Results with nickel

Conditions as in Figure 7

In reality these curves are the same as the 100 per cent anode efficiency curves inserted in the preceding figures, except that in these curves the currents are expressed in amperes, whereas in the other cases the equivalent weight of metal corresponding has been used.

In Figure 10 is shown the appearance of corroded copper anode surfaces - $(a)$ a partially immersed anode corroded by the application of an external emf under ordinary conditions (that is, without the use of an oxygen atmosphere), and (b) an anode corroded similarly in an atmosphere of oxygen. It will be seen that in the former case the surface attack was uniform, whereas in the latter case it was not, the corrosive attack in a band across the anode surface adjacent to 
the oxygen atmosphere being distinctly different from that over the remainder of the corroded surface. In order to render the anodic attack more distinct for inspection and photographic purposes, the solution in these cases was that which is used for the "ferroxyl"corrosion test. This solution, in addition to sodium chloride (10 $\mathrm{g} /$ /iter), contained a small amount of potassium ferricyanide ( $2 \mathrm{~g} /$ /iter) and enough agar-agar ( $4 \mathrm{~g} /$ /iter) to cause the solution to "jell" upon cooling. A reddish-brown coloration (cupric ferrocyanide) on the anode, which is produced by the reaction with the ferricyanide of the copper as it passes into solution, gives a record of the uniformity in distribution of the corrosive attack.

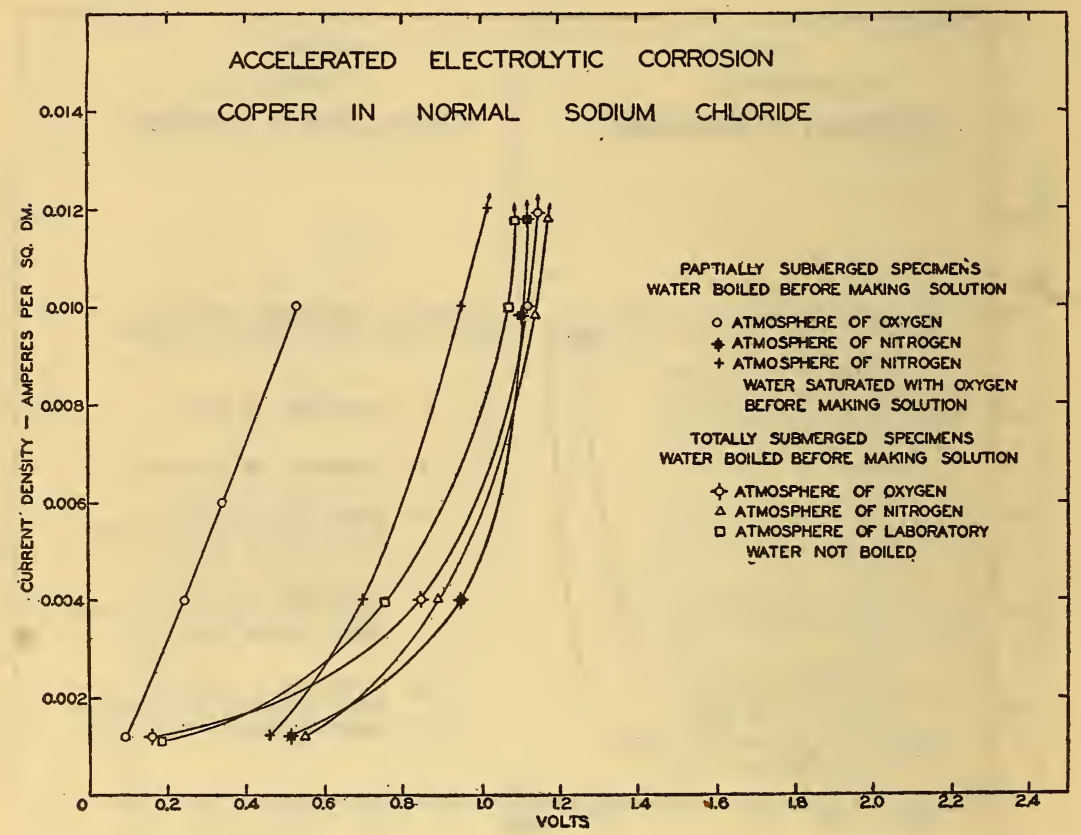

Figure 9.-Typical current density-potential curves for copper

A corresponding band across the cathode surface, just below the surface of the liquid, where the cathodic reaction was different from that over the remainder, was also observed when an oxygen atmosphere was used.

\section{DISCUSSION}

The importance of the rôle played by oxygen in submerged corrosion under ordinary conditions is generally recognized. Indeed, as stated by Evans, ${ }^{6}$ "Corrosion * * * by ordinary industrial waters, which are neutral or nearly neutral, requires the presence of oxygen." Advantage is taken of this fact, in a practical way, for the mitigation

\footnotetext{
- U. R. Evans, The Corrosion of Metals, p. 67; Edward Arnold_\& Co. (London); 1924.
} 
B. S. Journal of Research, RP101

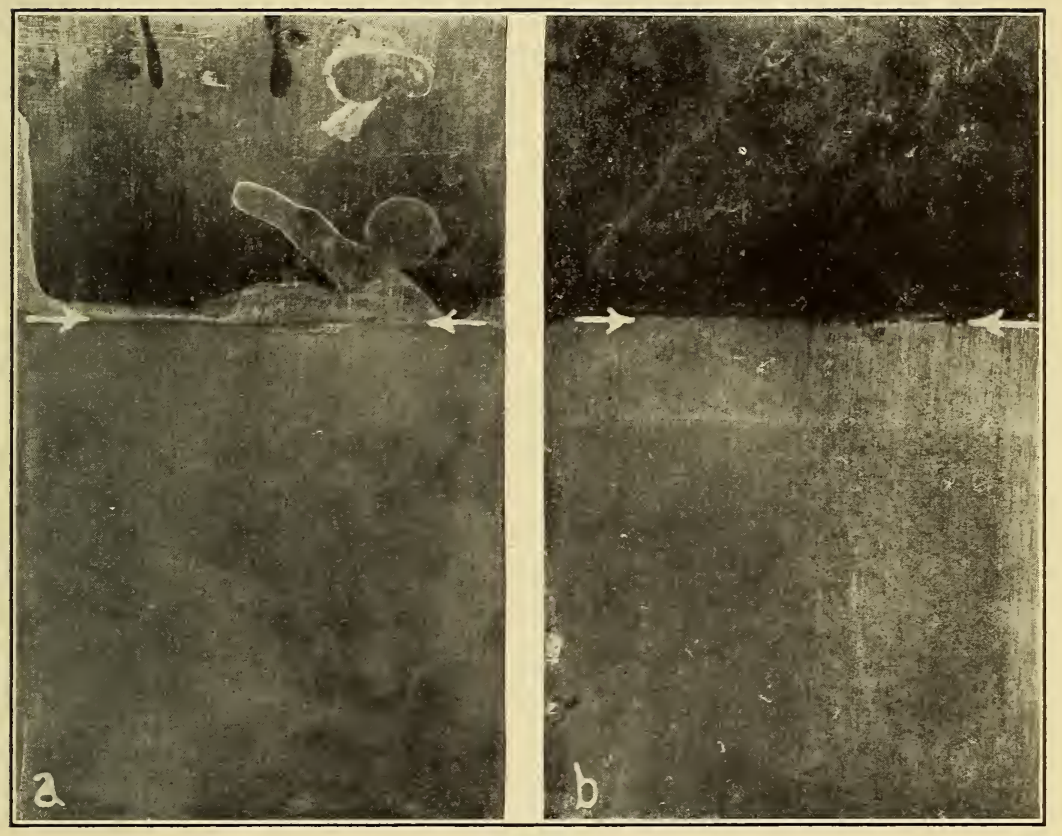

FIGURE 10.-Appearance of the anode surface of copper corroded electrolytically in sodium-chloride solution

(a) Under ordinary conditions; (b) with an overlying atmosphere of oxygen. The arrows indicate the "water line" 
or prevention of corrosion in water-heating systems and the like, by removing the oxygen from the water before it enters the system. There is very little information available as to the possible corresponding effect of oxygen when the corrosive attack is accelerated electrolytically. It is evident, however, from the foregoing results that the effect of the presence of oxygen on electrolytic accelerated corrosion may be very pronounced. Especially is this true for those conditions which most closely approach those of "natural corrosion"; that is, low anode losses (low current densities). This discussion relates largely to results of this kind; that is, anode losses below $0.20 \mathrm{~g}$ (per sq. dm 24 hours) for copper and below $0.10 \mathrm{~g}$ for nickel. The fact that the foregoing results show that the presence of oxygen reduces the potential required in order to produce a given rate of corrosion may be taken as indicating, in general, the reliability of the electrolytic corrosion test when applied under conditions corresponding to given types of exposure.

The difference in the general nature of the results obtained when oxygen was used and those when oxygen was excluded by means of nitrogen (fig. 7) is very striking. The reason for this difference in behavior which first occurs to one is the "depolarizing" action of oxygen in the one case and the lack of it in the other. The results in this case illustrate very clearly the fact previously mentioned that there is no single "standard" based upon the Faraday loss which may be used for comparison of these widely varying results. It will be noted that the curves showing 100 per cent anode efficiency for the runs made under these varying conditions differ quite decidedly among themselves. The agreement between the observed corrosion losses and the corresponding 100 per cent anode efficiency curve was better. In practically all cases, however, the observed losses were greater than the calculated ones. Any loss in weight greater than that corresponding to 100 per cent anode efficiency is evidence that all of the current contributing to the corrosive attack was not measured; that is, some current flowed from one part of the anode to another part of the same as a result of some nonuniformity either in the metal or solution. A necessary and fundamental assumption of the electrolytic corrosion testing method is that a condition of uniformity does obtain. Except as these conditions are realized, the electrolytic test is "imperfect" and may be misleading. The present paper illustrates the importance and difficulty of fulfilling these conditions.

When oxygen was present in the solution its depolarizing action on the cathode surface may be considered as accounting, in part at least, for the greater corrosion losses which occurred as compared with the corresponding losses which occurred in solutions free from oxygen or containing only a relatively, small amount. In order to explain 
satisfactorily, however, the relatively high corrodibility when oxygen was used, particularly with specimens which were only partially submerged, it would seem that some additional cause must be sought. The effect of the uniform distribution of oxygen, which has been so ably demonstrated by Evans, appears to be an important factor in this regard.

As stated by Evans," "An essential condition for the setting up of electrochemical action is some departure from uniformity." Variations in the oxygen distribution in the chloride solution with respect to the metal strips immersed therein would result in a change in the potential of certain portions of the otherwise uniform metal surface with respect to the solution. That part in contact with an oxygenrich solution, such as the upper layer of the solution adjacent to an overlying oxygen atmosphere, would be cathodic in its behavior, whereas the portion of the same specimen bathed by solution having a lower oxygen content, anodic. This condition would, in itself, result in some electrolytic or corrosive action on a specimen.

A few experiments were carried out upon specimens of zinc, copper, and nickel in the manner described by Evans, to illustrate the fact that if two specimens of the same metal, connected electrically, are submerged at different depths in a solution so that one is exposed to more oxygen than the other, an emf is set up between the two. The metals, copper and nickel, when used in a sodium-chloride solution exposed to the atmosphere, behaved in the same manner as zinc under corresponding conditions, the behavior of which has been discussed in detail by Evans. ${ }^{8}$

The specimen near the surface of the solution hence, bathed by the solution of higher oxygen content was cathodic toward the one immersed deeper in the same solution; that is, in contact with a solution of lower oxygen content, as shown by potentiometer measurements as well as by use of a milliammeter. The measurement of the emf set up under these conditions is not at all a simple matter; the relatively high initial value observed rapidly drops as the experiment is allowed to proceed. Only qualitative tests were made to establish the fact that the behavior of the three metals was identical in their general behavior. Additional tests carried out under a bell jar and within an oxygen atmosphere showed that the magnitude of the emf set up was not greatly changed by the introduction of oxygen. The observations made on the uniformity of the corrosive attack of the anode (fig. 10) showed, however, that the area over which the differential-oxidation effect extended was decidedly increased by increasing the depth of the oxygen-rich surface layer of the solution.

7 U. R. Evans, The Corrosion of Metals, p. 68, Edward Arnold \& Co. (London); 1924.

8 U. R. Evans, The Corrosion of Metals, p. 72, Edward Arnold \& Co, (London); 1924, 
If the potential of an immersed specimen, as a whole, is changed with respect to the solution by the application of an external emfthat is, raised (made an anode) or lowered (made a cathode)-the other condition which is the result of the nonuniformity in the oxygen distribution in the solution would be expected to remain unaltered. In effect, some portions of that specimen which, as a result of the external emf, is made to act as anode of the cell would be expected to be more reactive (more anodic) than would result from the external emf alone and, likewise, portions of the cathode of the cell somewhat more strongly cathodic than otherwise. On the other hand, it might be expected that areas which would be less reactive and less cathodic than would be indicated by the applied emf would exist on the anode and cathode, respectively. A somewhat analogous case would be expected to result from the use of electrodes which were nonuniform initially; for example, the use of electrodes of the same kind of metal which had been cold-worked over a portion of its length.

It will be seen from the curves that the effect of the use of an oxygen atmosphere was more pronounced than that alone of oxygen dissolved in the solution. The results obtained with partially immersed specimens when an oxygen atmosphere was used were practically identical with those obtained when, in addition to the oxygen atmosphere, oxygen was dissolved in the solution. The use of nitrogen in the solution with an overlying atmosphere of oxygen did not very materially lower the effect, since the condition of nonuniformity still obtained. The slight lowering of the corrosive attack in this case can, perhaps, be attributed to a lessening of the cathodic depolarization.

The most pronounced effect attributable to nonuniformity of oxygen distribution occurred in those cases in which partially submerged specimens were used. Undoubtedly, however, this differential-oxidation condition also obtains to some extent for totally submerged specimens and will account for the relatively higher corrosion losses obtained in oxygen-containing solutions. The specimens, completely immersed, were, of necessity, held vertically in the solution, the upper edge being $0.5 \mathrm{~cm}$ below the surface. On such a specimen the effect of nonuniform oxygen distribution would be expected to be more noticeable than on one immersed horizontally.

The most puzzling case of the entire series is the one in which the solution, which contained oxygen, was enveloped by an atmosphere of nitrogen. (Fig. 7.) Under these conditions the corrosion behavior of copper in sodium chloride agreed more closely with that obtained when nitrogen was used in the solution than the other cases with oxygen. If it is assumed that the two principal factors to be considered are the degree of cathodic depolarization and the auxiliary 
electrolytic effect resulting from the nonuniformity within the solution with respect to oxygen, it would seem that the latter in this case for some reason is less effective than the former. A clear and fully satisfactory explanation for this particular case is not yet forthcoming.

Consider now the results obtained when the tests were carried out, under ordinary conditions; that is, without the use of oxygen or nitrogen. (Figs. 3 and 4.) The variations in the test procedure consisted in the use of partially and of totally submerged specimens in solutions made from gas-free (boiled) water and from water which had not been boiled. It is evident that, in these cases, the effect which has been attributed to nonuniformity of oxygen distribution was practically negligible for the metals copper and nickel. At least it was not detected by the means employed in the test. With a more reactive metal, such as zinc, this might not be the case. The effect resulting from using a gas-free solution in some of the tests was quite marked and of sufficient magnitude to indicate that this factor should be taken into account. If the accelerated electrolytic corrosion test is to be used as a means for determining the relative corrodibilities of different metals, all the tests should be made with the same kind of solution; that is, with respect to the presence or absence of oxygen in the solution.

\section{SUMMARY}

Accelerated electrolytic corrosion tests on sheet copper and nickel in a sodium-chloride solution have been carried out by a method which has been previously described. Both anode and cathode in any one test were of the same metal, and the corrosion or anode losses have been expressed in the form of loss-of-weight-potential curves, together with the corresponding 100 per cent anode efficiency curves computed in accordance with Faraday's laws for the current which resulted from the applied emf's. The conditions with respect to oxidation were varied by inclosing the electrolytic cell in an oxygen or a nitrogen atmosphere, as well as by using a gas-free solution or one through which oxygen or nitrogen had been passed. The specimens were completely immersed in some cases and only partially in others. The results of the tests may be summarized as follows:

1. In general, only for small anode losses (low current densities) did the results of accelerated electrolytic corrosion tests, carried out in the manner used here, seem to have any real significance for the various conditions investigated. For higher current densities the loss-of-weight-voltage curves agreed quite closely with one another. Incidentally, however, it may be pointed out that the behavior in 
electrolytic corrosion tests with low current densities would be expected to throw more light on the probable behavior under conditions of "natural" corrosion than would the behavior of the same metal with higher current density.

2 . The results obtained with partially immersed specimens, especially when an oxygen atmosphere was used, differed quite materially from those with totally immersed specimens, the anode losses in the first case being decidedly greater than when the specimen was completely immersed, other conditions remaining unaltered.

3. The corrosion loss of partially immersed anodes with an overlying oxygen atmosphere was considerably greater than the corrosion loss of a totally immersed anode under the same conditions, except for very low current densities. By using a nitrogen atmosphere above the solution or by saturating the solution with nitrogen the corrosion losses on both totally and partially immersed anodes were reduced considerably; that is, a higher external emf was necessary in order to cause the same loss as occurred with lower voltages under the other conditions. The results confirm the reliability of the electrolytic corrosion test, at least to the extent of showing that the presence of oxygen reduces the potential required to produce a given rate of corrosion. Both copper and nickel behaved similarly in all respects under the different test conditions, the difference being more pronounced in the case of copper, however.

4. The differential-oxidation effect on a metal immersed in a solution, whereby the potential with respect to the solution of that part of the specimen which does not have free access to the air (oxygen) is rendered distinctly different (made anodic) from the potential of those parts of the same specimen having greater access to the air (cathodic), is believed to be associated with the relatively high corrosion losses obtained in many of the tests. This effect of differential oxidation originates within the "cell" and is to be considered as additional to that resulting from the application of an external emf to the cell. Examination of the corroded anode surface showed a distinct nonuniformity in the anode surface attack in those cases in which the differential-oxidation factor had been intensified (partial immersion in a solution with an overlying atmosphere of oxygen). The effect of oxygen in the solution as a "depolarizer" of the cathode surface and the absence of this effect when oxygen was excluded will account in large measure for the relatively low corrosion losses obtained when nitrogen was used. In practically all cases the observed corrosion losses were greater than those shown by the 100 per cent anode efficiency curve for corresponding conditions. It is obvious, therefore, that the entire current responsible for these losses was not measured. Currents set up as a result of potential differ$64338^{\circ}-29-4$ 
ences resulting from some nonuniformity, either in the metal surface or in the solution, by reason of variations in oxygen concentration may account for these greater corrosion losses.

5 . On the basis of the results of the present study, the accelerated electrolytic corrosion test can not, as yet, be considered a routine corrosion test. It is believed, however, that as a means of studying corrosion phenomena its value has been clearly demonstrated by the results of the study reported herein.

Washington, May 9, 1929. 